\title{
Association between Maternal Adverse Childhood Experiences and Attention-Deficit/Hyperactivity Disorder in the Offspring: The Mediating Role of Antepartum Health Risks
}

\author{
Duk-Soo Moon ${ }^{1 *}$, Su-Jeong Bong ${ }^{2 *}$, Bung-Nyun $\mathrm{Kim}^{3}$, and Na Ri Kang ${ }^{1,4}$ \\ ${ }^{1}$ Department of Psychiatry, Jeju National University Hospital, Jeju, Korea \\ ${ }^{2}$ Medical Course, Jeju National University School of Medicine, Jeju, Korea \\ ${ }^{3}$ Department of Psychiatry, Seoul National University School of Medicine, Seoul, Korea \\ ${ }^{4}$ Department of Psychiatry, Jeju National University School of Medicine, Jeju, Korea
}

\begin{abstract}
Objectives: This study aimed to examine the effect of maternal adverse childhood experiences (ACEs) on the attention-deficit/hyperactivity disorder (ADHD) symptoms in the offspring and to examine the mediating role of antepartum health risk on the intergenerational transmission of maternal ACEs.

Methods: The participants consisted of 461 mother-child dyads. Mothers completed the ACEs questionnaire and Diagnostic Predictive Scales. Multivariate logistic regression analysis was used to estimate the risk of ADHD symptoms in the offspring of mothers with ACEs and the mediating effect of antepartum health risks by path analysis.

Results: In all, 35.4\% ( $\mathrm{n}=163)$ had at least one maternal ACE, and 11.1\% ( $\mathrm{n}=51)$ had three or more. Compared to the non-ADHD symptom group, the group of offspring with ADHD symptoms showed a significant association with maternal ACE score ( $\mathrm{p}<0.001)$ and antepartum health risks $(\mathrm{p}<0.001)$. Multivariate analysis further showed a significant association between the sum of maternal ACEs [odds ratio $(\mathrm{OR})=1.264,95 \%$ confidence interval $(\mathrm{CI})=1.060-1.516, \mathrm{p}=0.009]$, antepartum health risks $(\mathrm{OR}=1.236,95 \% \mathrm{CI}=1.036-1.475, \mathrm{p}=$ 0.019), and ADHD symptoms in the offspring. In the mediation model in which the mother's ACE score affected the offspring's ADHD symptoms, partial mediation through antepartum health risks was found to be significant ( $\mathrm{B}=0.041,95 \% \mathrm{CI}=0.011-0.124)$.

Conclusion: Maternal ACEs are significantly related to the incidence of ADHD symptoms in the offspring and antepartum health risks exert an indirect effect. These findings suggest that maternal ACEs have a negative impact on the offspring's brain development through intergenerational transmission, resulting in neurodevelopmental disorders such as ADHD.
\end{abstract}

Key Words: Adverse childhood experiences; Attention-deficit/hyperactivity disorder; Antepartum health risks.

Received: October 16, 2020 / Revision: November 25, 2020 / Accepted: November 27, 2020

Address for correspondence: Na Ri Kang, Department of Psychiatry, Jeju National University School of Medicine, 15 Aran 13-gil, Jeju 63238, Korea

Tel: +82-64-717-1850, Fax: +82-64-717-1849, E-mail: ilnaree@hanmail.net

*These authors contributed equally to this work.

\section{INTRODUCTION}

Adverse childhood experiences (ACEs) are known to affect neurodevelopment and the endocrine and immune systems, thus increasing cognitive, social, and emotional dysfunctions [1-3]. Furthermore, people with many ACEs have many physical or mental problems and a high premature mortality rate [4]. A recent meta-analysis reported that the impact of ACEs is transmitted down to generations and is closely linked to the risk of ACEs (violence, mental illness, substance abuse) in the next generation [3], and parents' ACEs have an adverse im-

This is an Open Access article distributed under the terms of the Creative Commons Attribution Non-Commercial License (https://creativecommons.org/licenses/by-nc/4.0) which permits unrestricted non-commercial use, distribution, and reproduction in any medium, provided the original work is properly cited. pact on the socioemotional growth of their children [5]. In particular, the presence of four or more ACEs in parents was associated with hyperactivity in their offspring [6].

Attention-deficit/hyperactivity disorder (ADHD) is a neurodevelopmental disorder that is understood as a brain maturational delay caused by an interaction among various factors, including, biological, environmental, and psychosocial risk factors [7]. Because antepartum health risks can have a detrimental impact on fetal brain development, studies have investigated antepartum health risks associated with ADHD. Currently, the identified antepartum health risks associated with ADHD include maternal smoking, antepartum stress, preterm birth, low birth weight, and pregnancy-related complications $[8,9]$. 
According to previous studies, ACEs are positively correlated with antepartum health risks, and people with ACEs have difficulty controlling their emotions and seek strong compensatory strategies to mitigate negative emotions during pregnancy. This alters the neurobiological circuit and increases the risk of binge drinking during pregnancy [10], and mothers with a high number of ACEs exhibited a high risk for pregnancy diabetes, preeclampsia, low birth weight infants, and short gestation period [5]. Furthermore, chronic stress caused by ACEs elevates risky health behaviors, such as smoking, drinking, and binge eating, which are likely to lead to adverse birth outcomes, such as miscarriage and preterm birth [11].

While studies on the mediators of the impact of maternal ACEs on offspring's ADHD are lacking, based on previous findings, maternal ACEs are likely to increase antepartum health risks and thus have an adverse impact on children's neural development. Thus, this study aimed to investigate the effects of maternal ACEs and antepartum health risks on the incidence of ADHD in the offspring and the mediating role of antepartum health risks in the intergenerational transmission of maternal ACEs in the form of ADHD.

\section{METHODS}

\section{Participants and methods}

This study involved mothers and their children aged 6-18 years who had continuously been attending school for at least 6 months in Jeju Island. Data were collected using a questionnaire, which was distributed and retrieved by mental health staff trained on the background of the study and contents of the questionnaire. A total of 461 participants were enrolled, and written informed consent was obtained from the mothers and their offspring. Data were collected from September to December 2017. This study was approved by the Institutional Review Board of Jeju National University Hospital (2018-08-004).

\section{Instruments}

\section{Adverse childhood experiences questionnaire}

The ACEs questionnaire retrospectively surveys adverse experiences in childhood before the age of 18 years, including child abuse (emotional abuse, physical abuse, sexual abuse), child neglect (physical neglect, emotional neglect), and dysfunctional family environment (domestic violence, parents' divorce or separation, family member's substance abuse, mental illness, and incarceration) [12]. A "yes" response is scored 1 point, and "no" response, 0 . The total score is used to indicate the degree of ACE exposure [3]. ACEs are ana- lyzed in various ways, but the total score is used in most cases [13]. In the present study, the cumulative total score was classified into $0,1,2$, and $\geq 3$, and to examine the impact of each type of ACE on children's mental health problems, the scores for 3 domains of child abuse, child neglect, and household dysfunction were used.

\section{Diagnostic Interview Schedule for Children \\ Diagnostic Predictive Scales}

In this study, ADHD symptoms were assessed using the Diagnostic Predictive Scales (DPS). It is the primary screening tool of the Diagnostic Interview Schedule for Children (DISC)IV, a structured interview tool based on the DSM-IV criteria. The DPS for parents is a self-reported tool that consists of 92 questions about the symptoms of a mental disorder of the child in the past year and eight questions about mood or behavioral problems the child has at home, school, or with friends. DPS is a brief scale but can be effectively used for DISCIV diagnosis, with a high reliability [14].

A previous epidemiological survey on Korean children and adolescents [15] added 33 items to the DISC-IV but not the DPS (selective mutism, eating disorder, elimination disorder, posttraumatic stress disorder, tic disorder, cigarette, pica, and trichotillomania) and removed eight items related to general mood and behavior problems. The same tool was used in this study. We used the cutoff proposed in the study by Cubo for each disorder [16].

\section{Assessment of antepartum health risks}

The following 12 items pertaining to antepartum health risks are examined: physical problems, namely problems with fetal location, premature rupture of membranes, severe vaginal bleeding, and mild vaginal bleeding; rubella; preeclampsia; seizures in pregnancy; emotional problems requiring treatment, serious family problems; accidents or injuries requiring treatment; constipation; low back pain; perineal infection; heartburn; and severe psychological stress. These items about antepartum health risks were drawn from obstetric evaluation tools [17,18]. In this study, a "yes" response was given 1 point, and the total score was used to represent antepartum health risks.

\section{Statistical analysis}

Participants' demographic factors were analyzed using descriptive statistics and frequency analysis according to the total ACE score. The differences in maternal demographic factors, maternal antepartum health risk, and maternal ACEs according to the offspring's ADHD symptoms were analyzed using independent sample t-tests and chi-squared tests. Multiple logistic regression was performed for two models (ante- 
partum health risk and ACE; Model 1: ACE total score, Model 2: ACE score for three domains) to identify the significant predictors of ADHD. The mediating effects were analyzed using Model 4, which is used to test simple mediation models, from the 76 statistical models in the PROCESS macro for SPSS proposed by Hayes [19]. The significance of the indirect effects, direct effects, and total effects were analyzed using bootstrapping, with 5000 samples and 95\% confidence intervals (CIs). The mediating effects were deemed statistically significant if 0 was not included in the $95 \%$ CI. Data were analyzed using SPSS v.18 (SPSS Inc., Chicago, IL, USA) and Process Macro for SPSS v.3.4 software, and statistical significance was set at $\mathrm{p}<0.05$.

\section{RESULTS}

\section{Participants' general characteristics}

The mean age of the 461 participants was $42.79 \pm 5.68$ years; further, 275 participants had graduated from high school or higher (59.7\%), and 258 (56.0\%) were in the middle class in terms of their socioeconomic status; $35.4 \%(n=163)$ had at least one ACE, and $11.1 \%(\mathrm{n}=51)$ had at least three ACEs. The mean antepartum health risk score was $0.77( \pm 1.30)$. The mean age of the children was 13.26 years $( \pm 3.91)$, with $38.6 \%(n=178)$ male and $61.4 \%(\mathrm{n}=283)$ female. A total of 205 children were in elementary school (44.5\%), while 256 were in middle school or high school (55.5\%) (Table 1).

\section{Comparison of sociodemographic factors, maternal ACE, and antepartum health risks nonbetween the ADHD symptom group and control group}

Among the offspring, 13.7\% ( $\mathrm{n}=64)$ had ADHD symptoms. The ADHD symptom group was younger than the control group $(\mathrm{p}=0.001)$. The maternal antepartum health risk score was higher in the ADHD symptom group $(\mathrm{p}<0.001)$, with a greater percentage of mothers who had ACEs $(\mathrm{p}<0.001)(\mathrm{Ta}-$ ble 2). In the ADHD symptom group, 27 were male (42.2\%) and 37 were female $(57.8 \%)$, and there were no significant differences in the ACEs and antepartum health risk scores according to the child's sex.

\section{Maternal ACEs and antepartum health risks and child's ADHD symptoms}

Multiple logistic regression was performed to examine the impact of total maternal ACE score and maternal antepartum health risk on the incidence of ADHD symptoms (Model 1) and the impact of three types of maternal ACEs and antepartum health risk on the incidence of ADHD symptoms (Model 2). In Model 1, the odds ratio (OR) of the incidence of ADHD symptoms in the presence of antepartum health risks
Table 1. Demographic characteristics of the study sample $(n=461)$

\begin{tabular}{|c|c|}
\hline \multicolumn{2}{|l|}{ Mother related variables } \\
\hline Maternal age & $42.79( \pm 5.68)$ \\
\hline \multicolumn{2}{|l|}{ Maternal education level } \\
\hline High (> high school) & $275(59.7)$ \\
\hline Middle (high school) & $158(34.3)$ \\
\hline Low (<middle school) & $16(3.5)$ \\
\hline Missing value & $12(2.6)$ \\
\hline \multicolumn{2}{|l|}{ Socioeconomic status } \\
\hline High & $135(29.3)$ \\
\hline Middle & $258(56.0)$ \\
\hline Low & $59(12.8)$ \\
\hline Missing value & $9(2.0)$ \\
\hline \multicolumn{2}{|l|}{ Total ACE score $(0-10)$} \\
\hline 0 & $298(64.6)$ \\
\hline 1 & $66(14.3)$ \\
\hline 2 & $46(10.0)$ \\
\hline$\geq 3(3-8)$ & $51(11.1)$ \\
\hline Antepartum health risks & $0.77( \pm 1.30)$ \\
\hline \multicolumn{2}{|l|}{ Children related variables } \\
\hline \multicolumn{2}{|l|}{ Sex } \\
\hline Male & $178(38.6)$ \\
\hline Female & $283(61.4)$ \\
\hline Age & $13.26( \pm 3.91)$ \\
\hline \multicolumn{2}{|l|}{ Education level } \\
\hline Elementary school & $205(44.5)$ \\
\hline Middle school and above & $256(55.5)$ \\
\hline
\end{tabular}

Data are presented as mean \pm standard deviation or $\mathrm{n}(\%)$. SD: standard deviation, ACEs: adverse childhood experiences

after adjusting for socioeconomic status, maternal age and education level, and child's sex and age was 1.264 (95\% CI= $1.060-1.516, \mathrm{p}=0.009$ ) and the OR of total maternal ACE score predicting the incidence of ADHD symptoms was 1.236 (95\% $\mathrm{CI}=1.036-1.475, \mathrm{p}=0.019)$. In Model 2, the OR of antepartum health risks in predicting incidence of $\mathrm{ADHD}$ symptoms was significant (OR: 1.275, 95\% CI=1.069-1.520, $\mathrm{p}=0.007$ ), but when the maternal ACE score was divided into three domains (child abuse, child neglect, household dysfunction), the ORs were not significant (Table 3).

\section{Effects of maternal ACEs on incidence of ADHD symptoms through antepartum health risks}

The effects of total maternal ACE score on the incidence of ADHD symptoms in the offspring through antepartum health risks were analyzed using mediation analysis $\left(\mathrm{R}^{2}=0.197\right.$, $\mathrm{F}=18.431, \mathrm{p}<0.001)$. The total maternal ACE score was significantly associated with the incidence of ADHD symptoms in the offspring $(\mathrm{B}=0.312, \mathrm{p}<0.001)$ and antepartum health risks $(B=0.186, p<0.001)$, and antepartum health risk was significantly associated with the incidence of $\mathrm{ADHD}$ symptoms $(\mathrm{B}=$ 
Table 2. Comparison of psychosocial variables between ADHD symptom group and non-ADHD symptom group

\begin{tabular}{|c|c|c|c|}
\hline & ADHD symptom $(n=64)$ & Non-ADHD symptom $(n=397)$ & p-value \\
\hline Maternal age & $41.70(6.68)$ & $42.98(5.48)$ & 0.154 \\
\hline Maternal education level & & & 0.130 \\
\hline High (> high school) & $45(72.6)$ & $230(59.4)$ & \\
\hline Middle (high school) & $16(25.8)$ & $142(36.7)$ & \\
\hline Low (<middle school) & $1(1.6)$ & $15(3.9)$ & \\
\hline Socioeconomic status & & & 0.442 \\
\hline High & $9(14.1)$ & $50(12.9)$ & \\
\hline Middle & $32(50.0)$ & $226(58.2)$ & \\
\hline Low & $23(35.9)$ & $112(28.9)$ & \\
\hline Children's sex (male) & $27(42.2)$ & $151(38.0)$ & 0.527 \\
\hline Children's age & $11.69(3.85)$ & $13.52(3.87)$ & 0.001 \\
\hline Children's education level & & & 0.002 \\
\hline Elementary school & $40(62.5)$ & $165(41.6)$ & \\
\hline Above middle school & $24(37.5)$ & $232(58.4)$ & \\
\hline Antepartum health risks & $1.30(1.19)$ & $0.69(1.30)$ & $<0.001$ \\
\hline Total ACE score & & & $<0.001$ \\
\hline 0 & $26(40.6)$ & $272(68.5)$ & \\
\hline 1 & $11(17.2)$ & $55(13.9)$ & \\
\hline 2 & $12(18.8)$ & $34(8.6)$ & \\
\hline+3 & 15 (23.4) & $36(9.1)$ & \\
\hline
\end{tabular}

Data are presented as mean \pm standard deviation or $\mathrm{n}(\%)$. ADHD: attention-deficit/hyperactivity disorder, ACEs: adverse childhood experiences

Table 3. Multivariable logistic regression analysis for offspring' ADHD symptoms by maternal ACEs and antepartum health risks

\begin{tabular}{|c|c|c|c|c|c|}
\hline & $B$ & SE & OR & $95 \% \mathrm{Cl}$ & p-value \\
\hline \multicolumn{6}{|l|}{ Model 1} \\
\hline Antepartum health risks & 0.234 & 0.090 & 1.264 & $1.060-1.516$ & 0.009 \\
\hline Total sum of maternal ACEs & 0.212 & 0.090 & 1.236 & $1.036-1.475$ & 0.019 \\
\hline \multicolumn{6}{|l|}{ Model 2} \\
\hline Antepartum health risks & 0.243 & 0.090 & 1.275 & $1.069-1.520$ & 0.007 \\
\hline \multicolumn{6}{|l|}{ Maternal ACE domains } \\
\hline Child abuse & 0.252 & 0.233 & 1.286 & $0.815-2.030$ & 0.280 \\
\hline Child neglect & 0.090 & 0.352 & 1.094 & $0.549-2.180$ & 0.799 \\
\hline Household dysfunction & 0.225 & 0.180 & 1.252 & $0.879-1.782$ & 0.212 \\
\hline
\end{tabular}

Adjusted socioeconomic status, maternal age, maternal education level, offspring's age and sex. Hosmer-Lemeshow goodness of fit $(p=0.969)$. ADHD: attention-deficit/hyperactivity disorder, ACEs: adverse childhood experiences, B: regression coefficient, SE: standard error of B, OR: odds ratio, Cl: confidence interval

0.220, $\mathrm{p}=0.009$ ) (Fig. 1). In the bootstrapping analysis to determine the significance of the direct and indirect effects of the ACE score, the total maternal ACE score had a significant direct effect on incidence of ADHD symptoms $(B=0.312,95 \%$ $\mathrm{CI}=0.151-0.472)$ and indirect effect through antepartum health risks $(\mathrm{B}=0.041,95 \% \mathrm{CI}=0.011-0.124)$ (Table 4).

\section{DISCUSSION}

Maternal ACEs and antepartum health risk are factors associated with $\mathrm{ADHD}$ symptoms in the offspring. In particular, maternal ACEs had significant indirect effects on the risk of ADHD symptoms in the offspring through antepartum health risks.

The antepartum health risks assessed in this study included not only major risk factors such as preeclampsia, premature rupture of membrane, and seizures, but also a wide range of physical symptoms, emotional problems, and stress during pregnancy. Our results showed that maternal antepartum health risks significantly affected the risk of ADHD symptoms in the offspring, similar to previous findings that ADHD diagnosis in children aged 6-12 years is associated with maternal stress life events during pregnancy [20] and that stressful life events during pregnancy were associated with the off- 


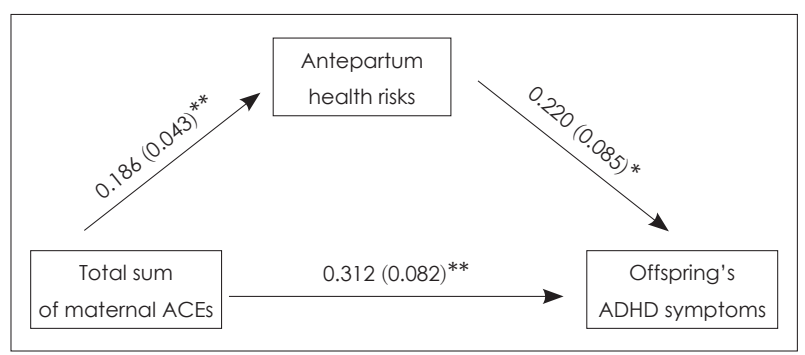

Fig. 1. Diagrams to test for mediation effect. Mediation model with a X (total sum of maternal ACEs), M (antepartum health risks), and $Y$ (offspring's ADHD symptoms). Standardized path coefficients and standard errors are presented. ${ }^{*} p<0.05,{ }^{* *} p<0.01$. ADHD: attention-deficit/hyperactivity disorder, ACEs: adverse childhood experiences.

Table 4. The bootstrap results for the indirect effect of maternal ACEs on offspring's ADHD symptoms via antepartum health risks

\begin{tabular}{lccc}
\hline Path & B & SE & $95 \% \mathrm{Cl}$ \\
\hline Direct effect & & & \\
Total sum of maternal ACEs $\rightarrow$ & 0.312 & 0.082 & $0.151-0.472$ \\
Offspring's ADHD symptoms & & & \\
Indirect effect & & & \\
Total sum of maternal ACEs $\rightarrow$ & 0.041 & 0.028 & $0.011-0.124$ \\
$\quad$ Antepartum health risks $\rightarrow$ & & & \\
Offspring's ADHD symptoms & & &
\end{tabular}

ADHD: attention-deficit/hyperactivity disorder, ACEs: adverse childhood experiences, B: regression coefficient, SE: standard error of $\mathrm{B}, \mathrm{Cl}$ : confidence interval

spring's ADHD symptoms in a prospective cohort study of 2900 participants [21].

The result that the cumulative ACE score, as opposed to the domains of ACE, has an adverse impact on the offspring's ADHD symptoms suggests that there is a dose-response relationship in the intergenerational transmission of mental health problems from parents to offspring, as observed in a previous study where cumulative risk was detrimental to child development [22].

Maternal ACE is significantly associated with antepartum health risk [11], and mothers with four or more ACEs have a two-fold increased biomedical risk during pregnancy [5]. Similar to our study, a previous study reported that maternal health risks in pregnancy and maternal ACEs led to adverse development outcomes in 12-month-old children [23]. Further, compared to children of mothers without childhood abuse experience, those of mothers with childhood abuse experience ACE showed a significantly high prevalence of ADHD, and adverse perinatal factors increased the risk of ADHD by $8.6 \%$ [24].

Prenatal stress has a direct impact on fetal neurodevelopment, such as delay in progenitor migration and reduction in forebrain proliferation [25]. Moreover, adverse environmental factors during pregnancy, such as prenatal stress, exposure to toxic substances, and infection, contribute to neurodevel- opmental impairment by inducing epigenetic changes related to gene expression in the fetus [26]. Genetic heritability accounts for $70-80 \%$ of ADHD cases [27], and such antepartum health risks are highly likely to contribute to the etiology of ADHD by increasing or facilitating the genetic liability of neurodevelopmental disorders [7,28]. In addition, ACEs, such as child abuse, lead to an imbalance in the hypothalamic-pituitary-thyroid axis and immune functions [1,2], which is in turn associated with ADHD, language and cognitive developmental delay, and externalizing behaviors in the offspring [29]. Therefore, maternal biological dysregulation during pregnancy is likely to serve as an important mechanism underlying the elevated risk of ADHD in the children.

The results of this study suggest that maternal ACEs can contribute to neurodevelopmental disorders such as ADHD by affecting fetal brain development through biological mediators during pregnancy. Similar to our findings, a prior study on autism reported that mothers with childhood abuse experience more commonly experienced gestational diabetes, preeclampsia, smoking and drinking behaviors during pregnancy, miscarriage, and preterm births, with a significantly higher risk of their children being autistic, than mothers without such experience [30].

This study has a few limitations. First, this study was conducted in a single center and considering that the prevalence of ACE varies across regions and cultures, the findings cannot be generalized, necessitating large-scale prospective studies. Second, we used a self-reported screening tool to diagnose ADHD instead of a clinical diagnostic or structured diagnostic tool based on the DSM-5. Third, we did not include all the possible antepartum health risks in our questionnaire. However, various antepartum physical and emotional problems were included in this study. Fourth, the ACE questionnaire asks the respondent whether he/she has undergone specific experiences and it is not a screening scale that determines the presence of clinical symptoms based on a cutoff. However, this scale is yet to be standardized for the South Korean population.

Nevertheless, this study is the first study to propose that maternal ACEs are associated with ADHD symptoms in their offspring through the mediation of antepartum health risks. Maternal ACEs can influence fetal neurodevelopmental, resulting in disorders such as ADHD via intergenerational transmission.

\section{CONCLUSION}

Maternal ACEs and antepartum health risks are both significant predictors of ADHD symptoms in the offspring, and maternal ACEs also indirectly influence the ADHD symp- 
toms through antepartum health risks. This suggests that in addition to the well-known impact of maternal ACEs on the offspring's emotional development, maternal ACEs negatively influence fetal brain development, contributing to neurodevelopmental disorders such as ADHD through intergenerational transmission. Future studies should examine the mediators and mechanisms through which maternal ACEs contribute to various neurodevelopment disorders other than ADHD in the offspring through intergenerational transmission.

\section{Acknowledgments}

This work was supported by a research grant from the Jeju National University Hospital (Grant No. 2018-08-004) Development Fund in 2018.

\section{Conflicts of Interest}

The authors have no potential conflicts of interest to disclose.

\section{Author Contributions}

Conceptualization: Na Ri Kang, Duk-Soo Moon, Bung-Nyun Kim. Formal analysis: Na Ri Kang, Su-Jeong Bong. Investigation: Na Ri Kang, Duk-Soo Moon, Bung-Nyun Kim. Writing-original draft: Su-Jeong Bong, Duk-Soo Moon. Writing-review \& editing: Duk-Soo Moon, Na Ri Kang.

\section{ORCID iDs}

Duk-Soo Moon https://orcid.org/0000-0001-7878-3410

Su-Jeong Bong https://orcid.org/0000-0002-4347-5589

Bung-Nyun Kim https://orcid.org/0000-0002-2403-3291

Na Ri Kang https://orcid.org/0000-0002-2086-0927

\section{REFERENCES}

1) Sinai C, Hirvikoski T, Nordström AL, Nordström $P$, Nilsonne $A$, Wilczek A, et al. Hypothalamic pituitary thyroid axis and exposure to interpersonal violence in childhood among women with borderline personality disorder. Eur J Psychotraumatol 2014;5:23911.

2) Fagundes CP, Glaser R, Kiecolt-Glaser JK. Stressful early life experiences and immune dysregulation across the lifespan. Brain Behav Immun 2013;27:8-12.

3) Hughes K, Bellis MA, Hardcastle KA, Sethi D, Butchart A, Mikton $\mathrm{C}$, et al. The effect of multiple adverse childhood experiences on health: a systematic review and meta-analysis. Lancet Public Health 2017;2:e356-e366.

4) Bellis MA, Hughes K, Leckenby N, Hardcastle KA, Perkins C, Lowey H. Measuring mortality and the burden of adult disease associated with adverse childhood experiences in England: a national survey. J Public Health (Oxf) 2015;37:445-454.

5) Madigan S, Wade M, Plamondon A, Maguire JL, Jenkins JM. Maternal adverse childhood experience and infant health: biomedical and psychosocial risks as intermediary mechanisms. J Pediatr 2017; 187:282-289.e1.

6) Schickedanz A, Halfon N, Sastry N, Chung PJ. Parents' adverse childhood experiences and their children's behavioral health problems. Pediatrics 2018;142:e20180023.

7) Barkley RA. Etiologies of ADHD. In: Barkley RA, editor. Attention-deficit hyperactivity disorder: a handbook for diagnosis and treatment. New York: The Guilford Press; 2015. p.356-390.

8) Rommel AS, James SN, McLoughlin G, Brandeis D, Banaschewski T, Asherson P, et al. Association of preterm birth with attention-defi- cit/hyperactivity disorder-like and wider-ranging neurophysiological impairments of attention and inhibition. J Am Acad Child Adolesc Psychiatry 2017;56:40-50.

9) Sciberras E, Mulraney M, Silva D, Coghill D. Prenatal risk factors and the etiology of ADHD - review of existing evidence. Curr Psychiatry Rep 2017;19:1.

10) Currie CL, Sanders JL, Swanepoel LM, Davies CM. Maternal adverse childhood experiences are associated with binge drinking during pregnancy in a dose-dependent pattern: findings from the All Our Families cohort. Child Abuse Negl 2020;101:104348.

11) Racine N, Madigan S, Plamondon A, Hetherington E, McDonald S, Tough S. Maternal adverse childhood experiences and antepartum risks: the moderating role of social support. Arch Womens Ment Health 2018;21:663-670.

12) Felitti VJ, Anda RF, Nordenberg D, Williamson DF, Spitz AM, Edwards V, et al. Relationship of childhood abuse and household dysfunction to many of the leading causes of death in adults. The Adverse Childhood Experiences (ACE) Study. Am J Prev Med 1998;14: 245-258.

13) Ford DC, Merrick MT, Parks SE, Breiding MJ, Gilbert LK, Edwards VJ, et al. Examination of the factorial structure of adverse childhood experiences and recommendations for three subscale scores. Psychol Violence 2014;4:432-444.

14) Lucas CP, Zhang H, Fisher PW, Shaffer D, Regier DA, Narrow WE, et al. The DISC Predictive Scales (DPS): efficiently screening for diagnoses. J Am Acad Child Adolesc Psychiatry 2001;40:443-449.

15) Cho SC, Go BJ, Kim BN, Kim JW, Shin MS, Yoo HI, et al. The 2005 Seoul child and adolescent mental health survey. Seoul: Child and Adolescent Mental Health Center;2006.

16) Cubo E, Velasco SS, Benito VD, Villaverde VA, Galín JM, Santidrián AM, et al. Psychometric attributes of the DISC predictive scales. Clin Pract Epidemiol Ment Health 2010;6:86-93.

17) Kim BN. Pregnancy, delivery and perinatal risk factors associated with symptoms of attention deficit/hyperactivity disorder: community study. J Korean Neuropsychiatr Assoc 2002;41:263-273.

18) Hong YM, Oh EY. An association of ADHD and their risk factors in pregnancy, delivery, perinatal stage. J Korean Neuropsychiatr Assoc 2000;39:1083-1092.

19) IsaacMustapha $M$. The art of effective research: a study utilizing multiple regression analysis (Hayes process macros for SPSS). London: SAGE Publications Ltd;2019.

20) Grizenko N, Shayan YR, Polotskaia A, Ter-Stepanian M, Joober R. Relation of maternal stress during pregnancy to symptom severity and response to treatment in children with ADHD. J Psychiatry Neurosci 2008;33:10-16.

21) Ronald A, Pennell CE, Whitehouse AJ. Prenatal maternal stress associated with ADHD and autistic traits in early childhood. Front Psychol 2011;1:223.

22) Evans GW, Li D, Whipple SS. Cumulative risk and child development. Psychol Bull 2013;139:1342-1396.

23) Racine N, Plamondon A, Madigan S, McDonald S, Tough S. Maternal adverse childhood experiences and infant development. Pediatrics 2018;141:e20172495.

24) Roberts AL, Liew Z, Lyall K, Ascherio A, Weisskopf MG. Association of maternal exposure to childhood abuse with elevated risk for attention deficit hyperactivity disorder in offspring. Am J Epidemiol 2018;187:1896-1906.

25) Stevens HE, Su T, Yanagawa Y, Vaccarino FM. Prenatal stress delays inhibitory neuron progenitor migration in the developing neocortex. Psychoneuroendocrinology 2013;38:509-521.

26) Kundakovic M, Jaric I. The epigenetic link between prenatal adverse environments and neurodevelopmental disorders. Genes (Basel) 2017;8:104.

27) Brikell I, Kuja-Halkola R, Larsson H. Heritability of attention-deficit hyperactivity disorder in adults. Am J Med Genet B Neuropsy- 
chiatr Genet 2015;168:406-413.

28) Hinshaw SP. Attention deficit hyperactivity disorder (ADHD): controversy, developmental mechanisms, and multiple levels of analysis. Annu Rev Clin Psychol 2018;14:291-316.

29) Ghassabian A, Henrichs J, Tiemeier H. Impact of mild thyroid hormone deficiency in pregnancy on cognitive function in children: lessons from the Generation R Study. Best Pract Res Clin Endocrinol Metab 2014;28:221-232.

30) Roberts AL, Lyall K, Rich-Edwards JW, Ascherio A, Weisskopf MG. Association of maternal exposure to childhood abuse with elevated risk for autism in offspring. JAMA Psychiatry 2013;70:508515. 